\title{
Magnetic resonance imaging texture analysis for the evaluation of viable ovarian tissue in patients with ovarian endometriosis: a retrospective case-control study
}

\author{
Dayong Lee, Hyun Jung Lee \\ Department of Obstetrics and Gynecology, School of Medicine, Kyungpook National University, Kyungpook National University Hospital, \\ Daegu, Korea
}

Background: Texture analysis has been used as a method for quantifying image properties based on textural features. The aim of the present study was to evaluate the usefulness of magnetic resonance imaging (MRI) texture analysis for the evaluation of viable ovarian tissue on the perfusion map of ovarian endometriosis.

Methods: To generate a normalized perfusion map, subtracted T1-weighted imaging (T1WI), T1WI and contrast-enhanced T1W1 with sequences were performed using the same parameters in 25 patients with surgically confirmed ovarian endometriosis. Integrated density is defined as the sum of the values of the pixels in the image or selection. We investigated the parameters for texture analysis in ovarian endometriosis, including angular second moment (ASM), contrast, correlation, inverse difference moment (IDM), and entropy, which is equivalent to the product of area and mean gray value.

Results: The perfusion ratio and integrated density of normal ovary were $0.52 \pm 0.05$ and $238.72 \pm 136.21$, respectively. Compared with the normal ovary, the affected ovary showed significant differences in total size $(p<0.001)$, fractional area ratio $(p<0.001)$, and perfusion ratio $(p=0.010)$ but no significant differences in perfused tissue area $(p=0.158)$ and integrated density $(p=0.112)$. In comparison of parameters for texture analysis between the ovary with endometriosis and the contralateral normal ovary, ASM $(p=0.004)$, contrast $(p=0.002)$, IDM $(p<0.001)$, and entropy $(p=0.028)$ showed significant differences. A linear regression analysis revealed that fractional area had significant correlations with ASM $\left(r^{2}=0.211\right)$, IDM $\left(r^{2}=0.332\right)$, and entropy $\left(r^{2}=0.289\right)$.

Conclusion: MRI texture analysis could be useful for the evaluation of viable ovarian tissues in patients with ovarian endometriosis.

Keywords: Endometriosis; Gadolinium; Magnetic resonance imaging; Ovary

\section{Introduction}

Endometriosis occurs in $6 \%$ to $10 \%$ of women of reproductive age, among whom approximately $30 \%$ to $50 \%$ are infertile $[1,2]$.
Therefore, for a patient with endometriosis who wants to conceive through ovarian preservation, the surgeon should use the appropriate surgical method to avoid destruction due to blood coagulation or destruction of the ovarian blood vessels to reduce damage

Received: May 27, 2021 • Revised: June 22, 2021 • Accepted: June 25, 2021

Corresponding author: Hyun Jung Lee, MD, PhD

Department of Obstetrics and Gynecology, School of Medicine, Kyungpook National University, Kyungpook National University Hospital, 130 Dongdeok-ro, Jung-gu, Daegu 41944, Korea

Tel: +82-53-200-5691•Fax: +82-53-200-5724•E-mail: hyunjunglee@knu.ac.kr 
to the residual ovarian tissue [3]. In addition to advances in surgical technology, various studies on clinical data have been attempted to find preoperative biomarkers useful in predicting the likelihood of pregnancy after surgery. Therefore, the need for preoperative imaging to assess ovarian tissue around endometriosis is raised to minimize damage to the ovarian reserve.

Recently, the increasing prevalence of ovarian endometriosis in young nulliparous women, who desire to preserve their ovarian function raised the necessity of magnetic resonance imaging (MRI) examination. Because the ovaries contain several stages of follicles, they are easily identified in most women's pelvic MRIs during the reproductive period $[4,5]$. T2-weighted images (T2WI) are the most useful sequences in the diagnosis of disorders in the ovaries and ovarian follicles in women of reproductive age [6]. The ovarian stroma shows a contrast enhancement similar to that of the myometrium, and its contrast enhancement pattern on T1-weighted images (T1WI) correlated with age and menopausal status [6]. Cystic follicles and functional ovarian cysts were found frequently and with variable appearances. Most cysts show discrete enhancement of the wall. In contrast to poorly perfused endometrial cysts, regardless of $\mathrm{T} 1$ high signal intensity, remnant ovarian tissue could appear well perfused. However, when the endometriosis involves the ovary, the typical magnetic resonance (MR) findings were displacement of the affected ovarian tissue by cystic lesions with high signal intensity on T1WI and T2WI $[7,8]$. However, hemosiderin deposit due to repeated bleeding within the endometriosis can cause a change in MR signal $[7,9,10]$. Ultimately, ovarian reserve can be defined as the quantity and quality of the remaining follicular pools that can grow in the presence of gonadotropin. Therefore, evaluating the state of the residual ovary in the image can be a biomarker for evaluating ovarian reserve.

In the present study, the effect of ovarian tissue enhancement was normalized by dividing the subtracted contrast enhancement T1WI by contrast enhancement T1WI. In addition, integrated density was defined as the sum of the values of the pixels in the image or selection. Texture characteristics are the intrinsic properties of an image and provide an efficient image classification system by which subtle alterations in the gray level distribution of an image can be detected [11]. We investigated the parameters for texture analysis in ovarian endometriosis, including angular second moment (ASM), contrast, correlation, inverse difference moment (IDM), and entropy, which is equivalent to the product of area and mean gray value. Consequently, the aim of this study was to evaluate the usefulness of texture analysis for the normalized perfusion map of pelvic MRI for predicting remnant ovarian tissues in patients with endometriosis.

\section{Methods}

Ethical statements: The Institutional Review Board (IRB) of Kyungpook National University Hospital (IRB No: KNUH 2017-06-012) approved the retrospective data collection and analysis. The need for informed consent was waived for the retrospective design of the study.

In this study, female patients who underwent ovarian cystectomy for endometrioma by surgery between January 2016 and December 2019 were included. After surgical cystectomy, histopathological examination was performed for final diagnosis in all the cases. To evaluate the diseased ovary in comparison with the normal ovary, the patients with endometriosis only on one side were included, and those with disease on the other side were excluded. Normal ovary was defined as that without endometriosis on preoperative ultrasonography and MRI.

All the examinations were performed on a 3.0-tesla MR machine (Skyra; Siemens Health Care, Erlangen, Germany). Before MRI examination, the patients underwent 6 hours of fasting followed by intramuscular administration of $20 \mathrm{mg}$ of hyoscine butylbromide (Buscopan; Boehringer Ingelheim, Ingelheim am Rhein, Germany) to inhibit bowel movement. In the study, a set of 8-channel phased-array body coils was applied. We performed the MRI examination using the same parameters for field of view, slice thickness, echo time, repetition time for T1WI, and contrast-enhanced T1WI acquisition to generate a normalized perfusion map. To obtain a normalized perfusion image, the non-contrast-enhanced image was subtracted from the contrast-enhanced image and then divided by the contrast-enhanced image. As a result, each pixel constituting the normalized perfusion map was theoretically coded from 0 for no perfusion to 1 for complete perfusion (Fig. 1). Each region of interest (ROI) was measured by specifying an outline of the ovaries affected by endometriosis at the level with the largest area of the remaining ovary and the normal contralateral ovary on T2WI by one radiologist who was blinded to the clinical information. To measure only the perfusion of the surviving ovarian tissue, pixels corresponding to the endometriotic cysts with a low perfusion state of $\leq 0.2$ and high perfusion state of $\geq 0.9$ were removed using the threshold technique. The fractional area ratio was defined as the ratio of tissue area with a perfusion ratio between 0 and 0.2 to the total area.

The area, mean and standard deviation of signal intensity, and integrated density of the affected ovary with endometrioma were compared with those of the normal ovary. Integrated density, as the sum of all pixel intensities in the ROI, indicates the total 

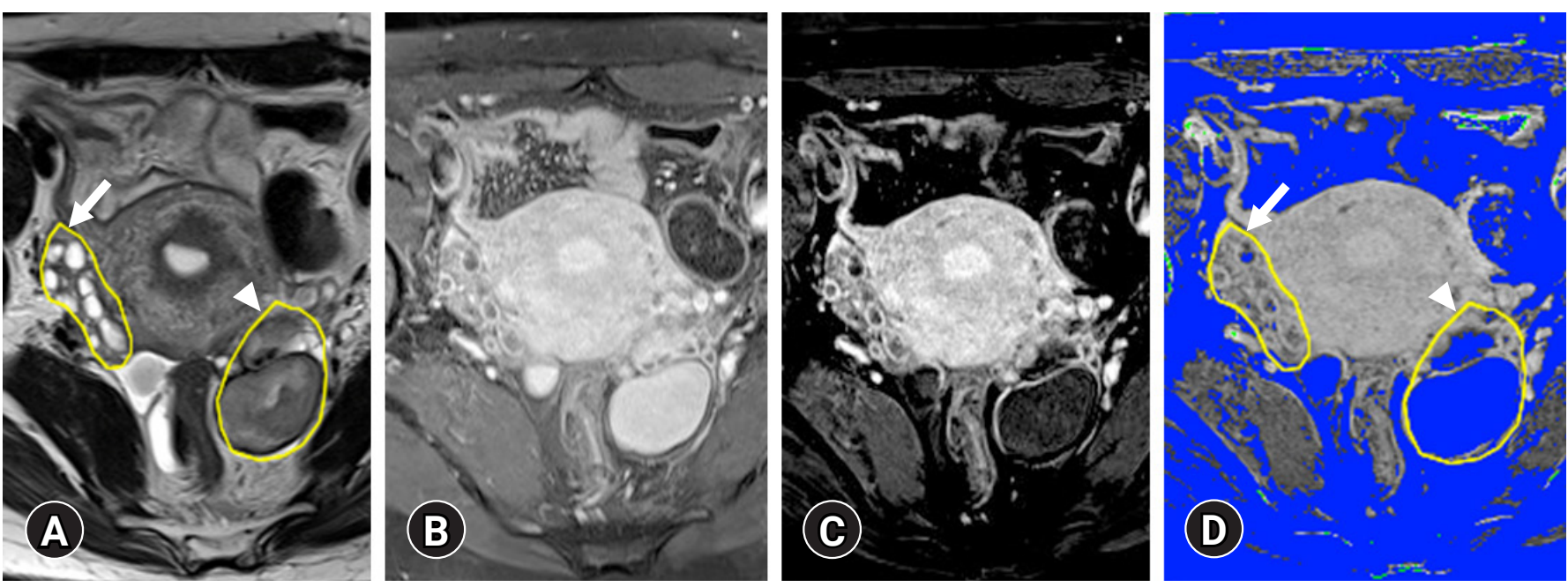

Fig. 1. (A) Region of interest (ROI) for normal ovary (arrow) and endometriosis (arrowhead) is defined on the T2-weighted image. (B, C) Division of the subtraction image by the contrast-enhanced T1-weighted image generates a normalized perfusion map. To measure only the perfusion of the surviving ovarian tissue, pixels corresponding to endometriotic cysts with a low perfusion state of 0.2 or less were removed using the threshold technique. (D) The saved ROI is applied to the normalized perfusion map.

amount of contrast enhancement effect in this study. The parameters included in the ASM, contrast, correlation, IDM, and entropy for the same ROI in the perfusion map, were calculated using the GLCM plugin using ImageJ (1.50i; National Institutes of Health, Bethesda, MD, USA; https://imagej.nih.gov/ij). Statistical analyses were performed using IBM SPSS version 23.0 for Windows (IBM Corp., Armonk, NY, USA). The paired $t$-test was used to examine the difference in numerical variables from those in the texture analysis. Statistical significance was set at a $p$-value of $<0.05$.

\section{Results}

This study included 25 patients with pathologically confirmed ovarian endometriosis after cystectomy. The mean age was $28.21 \pm 5.71$ years. Normal ovary was defined in all the patients. The normal ovaries showed variable-sized multiple cysts surrounded by solid ovarian stromal tissue on T2WI. At the level of the maximal diameter, normal ovaries showed 537.70 \pm 331.80 $\mathrm{mm}^{2}$ of area. Compared with the normal ovaries, ovaries affected by endometriosis had a higher number of pixels, with a perfusion ratio between 0 and 0.2 with poor perfusion due to the endometriotic cysts (Fig. 2). As a result, the perfused tissue area was $430.79 \pm 272.98 \mathrm{~mm}^{2}$ for the area of the tissue in the range of 0.2 to 0.9 . The ratio of the fractional area with a perfusion ratio of 0.2 to 0.9 to the total area was $0.89 \pm 0.11$. The perfusion ratio and integrated density of the normal ovary were $0.52 \pm 0.05$ and $238.72 \pm 136.21$, respectively.

Compared with the normal ovary, the total size $(p<0.001)$, fractional area ratio $(p<0.001)$, and perfusion ratio $(p=0.010)$ of the affected ovary showed significant differences, but the perfused tissue area $(p=0.158)$ and integrated density $(p=0.112)$ had no significant differences (Table 1 ).

In the comparison with the parameters for texture analysis between the ovary affected by endometriosis and the contralateral normal ovary, ASM $(p=0.004)$, contrast $(p=0.002)$, IDM $(p<0.001)$, and entropy $(p=0.028)$ showed significant differences (Table 2). However, all the cysts showed a low perfusion ratio on the normalized perfusion map (Fig. 3). Linear regression analysis revealed a significant coefficient of determination between the fractional area with ASM $\left(r^{2}=0.211\right), \operatorname{IDM}\left(r^{2}=0.332\right)$, and entropy $\left(r^{2}=0.289\right.$; Fig. 4). The standard coefficients of ASM, IDM, and entropy were $-0.459(p=0.021),-0.577(p=0.003)$, and 0.538 (0.006), respectively (Table 3 ).

\section{Discussion}

Until now, the effect of contrast enhancement on the evaluation of endometriosis using MRI has been shown to differentiate between other hemorrhagic adnexal lesions, luteal cysts, ovarian abscesses, or potentially malignant tumors $[12,13]$. However, this study focused on the role of contrast agents in the evaluation of adjacent surviving ovarian tissue invaded by endometriosis. The perfusion map could be used for the analysis of viable tissue within this subset of cystic ovarian masses according to the severity of endometriosis for preoperative evaluation [14-16].

In the present study, the total perfusion ratio of the normal ovary ranged from 0.2 to 0.9 as compared with that of cysts caused by endometriosis, which was $<0.2$ [17]. In this study, we suggest inte- 


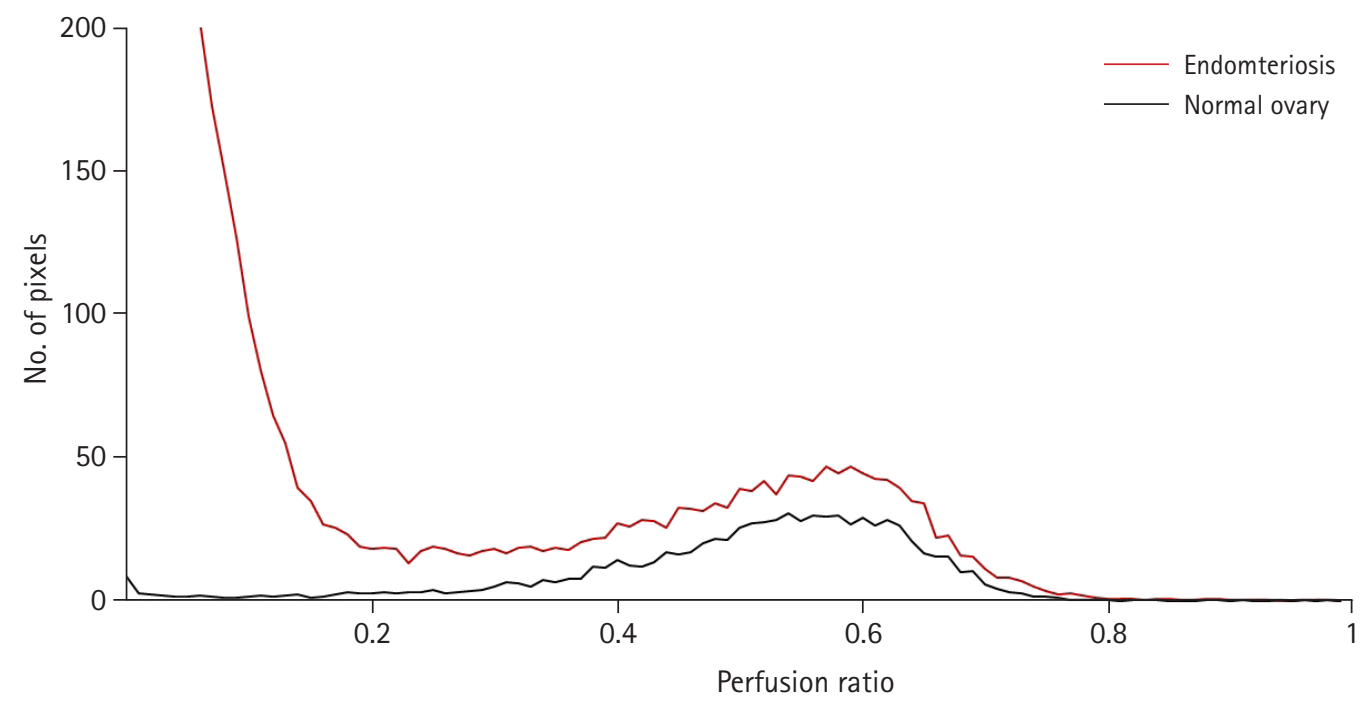

Fig. 2. Comparison of the number of pixels for the perfusion ratio between normal ovary (black line) and affected ovary by endometriosis (red line).

Table 1. Comparison of parameters of normalized perfusion map between affected ovary by endometriosis and contralateral normal ovary

\begin{tabular}{lccc}
\hline Parameter & Affected ovary by endometriosis & Contralateral normal ovary & $p$-value \\
\hline Total area $\left(\mathrm{mm}^{2}\right)$ & $2,050.40 \pm 1,067.99$ & $537.70 \pm 331.80$ & $<0.001$ \\
Perfused tissue area $\left(\mathrm{mm}^{2}\right), 0.2<$ perfusion ratio $<0.9$ & $550.43 \pm 270.22$ & $430.79 \pm 272.98$ & 0.158 \\
Fractional area ratio ${ }^{\text {b) }}$ & $0.33 \pm 0.19$ & $0.89 \pm 0.11$ & $<0.001$ \\
Perfusion ratio within perfused tissue area & $0.49 \pm 0.06$ & $0.52 \pm 0.05$ & 0.010 \\
Integrated density & $290.25 \pm 150.83$ & $238.72 \pm 136.21$ & 0.112 \\
\hline
\end{tabular}

Values are presented as mean \pm standard deviation.

a) Paired $t$-test. ${ }^{b}$ Ratio of tissue area with perfusion ratio between 0 and 0.2 to the total area.

Table 2. Comparison of histogram analysis between affected ovary by endometriosis and contralateral normal ovary

\begin{tabular}{lccc}
\hline Variable & Affected ovary by endometriosis & Contralateral normal ovary & $p$-value \\
\hline Angular second moment & $0.10 \pm 0.15$ & $0.01 \pm 0.03$ & 0.004 \\
Contrast & $779.96 \pm 396.83$ & $1,040.45 \pm 559.94$ & 0.002 \\
Correlation & $0.00062 \pm 0.00175$ & $0.00066 \pm 0.00154$ & 0.440 \\
Inverse difference moment & $0.31 \pm 0.18$ & $0.14 \pm 0.08$ & $<0.001$ \\
Entropy & $6.40 \pm 1.53$ & $7.10 \pm 0.75$ & 0.028 \\
\hline
\end{tabular}

Values are presented as mean \pm standard deviation.

a) Paired t-test.

grated density modified by area instead of pixel number. Although the further clinical study is needed, integrated density could be a potential biomarker of remnant ovarian tissue. In the present study, severe endometriotic invasion showed a smaller area with a moderate perfusion ratio similar to that of the ovary, which suggests that the perfusion map could predict remnant ovarian preserve. However, it is difficult to discriminate between the infiltration by the cyst accompanied by inflammatory changes due to endometriosis and the surrounding surviving ovarian tissue. Our results showed an increased pixel count at perfusion ratios between 0.4 and 0.9 in endometriosis, which suggests an increase in inflammatory changes in endometriosis and cystic lesions. These results may suggest that assessment of the degree of ovarian involvement may be helpful in determining the preoperative grade $[14,15]$. In this study, cysts were subtracted from the ovaries affected with endometriosis, and the remaining surviving ovarian tissues were compared with normal ovaries. The remainder after excluding the cyst site is presumed to be the changes caused by endometriosis, including inflammation and neovascularization, which will affect the texture analysis. 


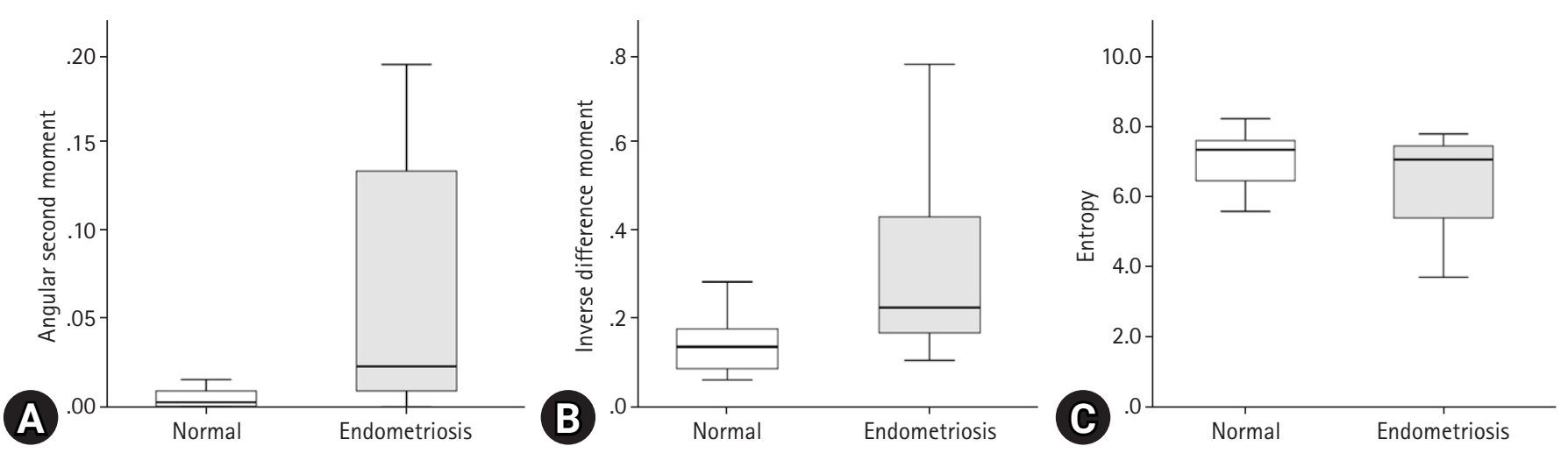

Fig. 3. Comparison of texture analysis parameters, including $(A)$ angular second moment, $(B)$ inverse difference moment, and (C) entropy, between affected ovary by endometriosis and the contralateral normal ovary.
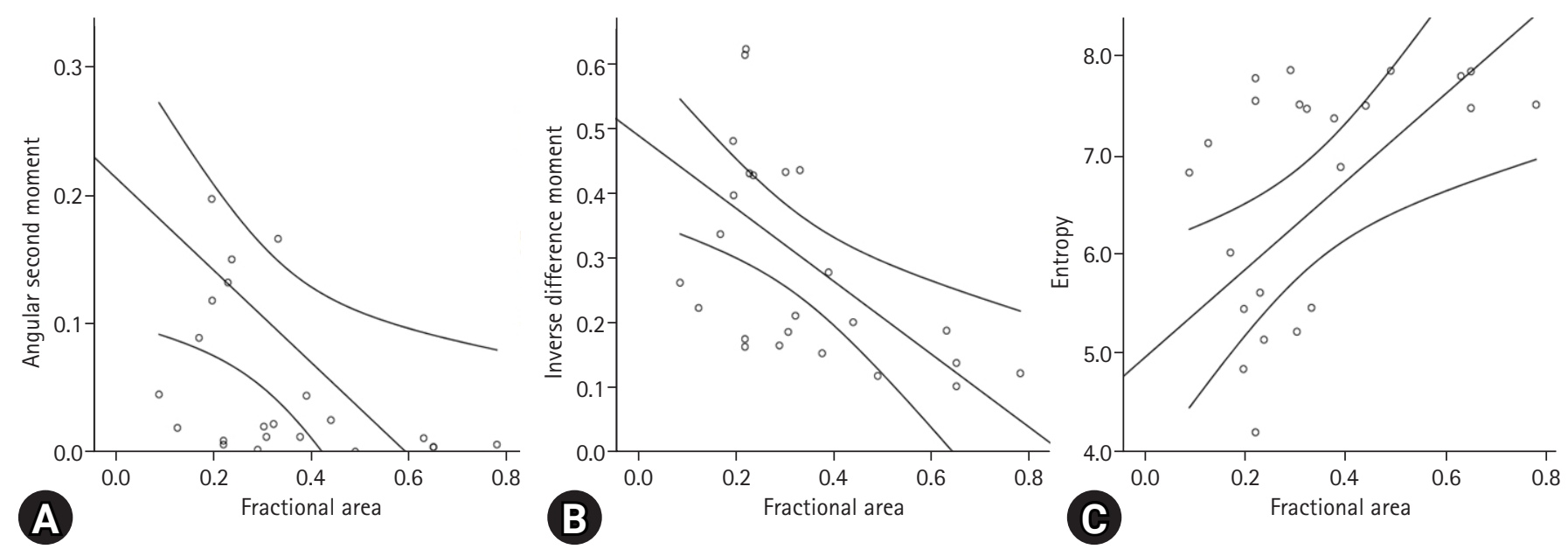

Fig. 4. Linear regression analysis of the parameters for texture analysis, including (A) angular second moment, (B) inverse difference moment, and $(\mathrm{C})$ entropy, of the affected ovary according to fractional area.

Table 3. Linear regression analysis between parameters of texture analysis with fractional area of ovarian tissue affected by endometriosis

\begin{tabular}{|c|c|c|c|c|c|c|}
\hline \multirow{2}{*}{ Model } & \multicolumn{2}{|c|}{ Unstandardized coefficient } & \multirow{2}{*}{ Standardized coefficient $\beta$} & \multirow{2}{*}{$t$} & \multirow{2}{*}{$r^{2}$} & \multirow{2}{*}{$p$-value } \\
\hline & $\beta$ & Standard error & & & & \\
\hline Angular second moment & -0.359 & 0.145 & -0.459 & -2.478 & 0.211 & 0.021 \\
\hline Inverse difference moment & -0.567 & 0.168 & -0.577 & -3.384 & 0.332 & 0.003 \\
\hline Entropy & 4.430 & 1.448 & 0.538 & 3.061 & 0.289 & 0.006 \\
\hline
\end{tabular}

${ }^{\text {a) }}$ Paired $t$-test.

Texture analysis has been used as a method for quantifying image texture, which is concerned with the description of the image properties based on textural features [18]. Texture analysis of medical images has expanded its applications to segmentation of specific anatomical structures, detection of lesions, differentiation, and prognosis of pathological and healthy tissues [19]. ASM, also called uniformity or energy, represents image homogeneity. A high ASM value represents good homogeneity of the image and remarkable similarity of pixels. The IDM score implies the local ho- mogeneity of the image. A high IDM score indicates uniformity in the gray level of the image. Entropy refers to the amount of image information required for image compression. The loss of image information or message can be measured by entropy. Texture analysis has been applied in various clinical areas and imaging methods but rarely applied in the evaluation of gynecological diseases, especially endometriosis, using MRI. Recently, histological analysis has been used to distinguish between cystic lesions of endometriosis and hemorrhagic cysts on MRI; therefore, images of selected le- 
sions were from T2WI [20]. However, the present study was conducted to compare infected endometrial tumors with normal ovaries; therefore, texture analysis was performed using a perfusion map based on contrast-enhanced T1WI.

However, this study has several limitations. First, the number of enrolled patients was too small for comprehensive understanding of the relationship between imaging results and clinical outcomes. For the validation of usefulness of texture analysis for prediction ability for pregnancy, a larger study population is mandatory. Second, the acquisition of perfusion maps requires that a trained radiologist strictly adheres to a specific protocol for obtaining an accurate image. Despite the need to create an image subtracted from correct pre- and postcontrast images, risk of disturbance caused by the patient's voluntary or involuntary movements remain $[21,22]$. Third, each ROI measurement was performed by a single observer, and the inter- and intraobserver variations were not assessed. However, these variations are expected to be small and barely affect the results of whole-volume measurements.

All the measurements and calculations after ROI measurement are made in the image postprocessing application, so these changes are limited and have little effect on texture analysis. Finally, the texture analysis result may vary depending on the image acquisition parameters, including image resolution, field size, and heterogeneity. Some of the features required to describe the structure of the tissue being examined can reflect the uneven sensitivity of the scanner, which can lead to inadequate description of the tissue.

This study focused on the role of texture analysis on perfusion imaging in patients with ovarian endometriosis. Normalized perfusion maps showed the extent of invasion of ovarian endometriosis to adjacent remnant ovarian tissue. The parameters of the perfusion map, including the perfusion ratio and integrated density in the residual ovary represented by the region with a perfusion ratio of 0.4 to 0.9 , showed a correlation with the degree of endometrial invasion. In conclusion, texture analysis for the perfusion map of pelvic MRI could be useful for revealing the extent of endometrial invasion and viable remnant ovarian tissue. Until now, texture analysis of perfusion maps of pelvic MRI has been studied very rarely and may be a promising tool for preoperative evaluation of residual ovarian tissue in patients who wish to preserve their ovaries during surgical treatment.

\section{Notes}

\section{Conflicts of interest}

No potential conflict of interest relevant to this article was reported.

\section{Author contributions}

Conceptualization, Formal analysis, Visualization, Resources, Software, Supervision: HJL; Project administration: DL; Data curation, Methodology: all authors; Writing-original draft: all authors; Writing-review \& editing: all authors.

\section{ORCID}

Dayong Lee, https://orcid.org/0000-0003-4340-8180

Hyun Jung Lee, https://orcid.org/0000-0002-3942-405X

\section{References}

1. Yu JS, Rofsky NM. Dynamic subtraction MR imaging of the liver: advantages and pitfalls. AJR Am J Roentgenol 2003;180: 1351-7.

2. Hummelshoj L, Prentice A, Groothuis P. Update on endometriosis. Womens Health (Lond) 2006;2:53-6.

3. Working group of ESGE and WES; Saridogan E, Becker CM, Feki A, Grimbizis GF, Hummelshoj L, et al. Recommendations for the surgical treatment of endometriosis-part 1: ovarian endometrioma. Gynecol Surg 2017;14:27.

4. Messinger Y, Yanishevski Y, Avramis VI, Ek O, Chelstrom LM, Gunther R, et al. Treatment of human B-cell precursor leukemia in SCID mice using a combination of the investigational biotherapeutic agent B43-PAP with cytosine arabinoside. Clin Cancer Res 1996;2:1533-42.

5. Togashi K, Nakai A, Sugimura K. Anatomy and physiology of the female pelvis: MR imaging revisited.J Magn Reson Imaging 2001;13:842-9.

6. Outwater EK, Mitchell DG. Normal ovaries and functional cysts: MR appearance. Radiology 1996;198:397-402.

7. Woodward PJ, Sohaey R, Mezzetti TP Jr. Endometriosis: radiologic-pathologic correlation. Radiographics 2001;21:193-216.

8. Sugimura K, Okizuka H, Imaoka I, Kaji Y, Takahashi K, Kitao M, et al. Pelvic endometriosis: detection and diagnosis with chemical shift MR imaging. Radiology 1993;188:435-8.

9. Sanchez AM, Viganò P, Somigliana E, Panina-Bordignon P, Vercellini $\mathrm{P}$, Candiani $\mathrm{M}$. The distinguishing cellular and molecular features of the endometriotic ovarian cyst: from pathophysiology to the potential endometrioma-mediated damage to the ovary. Hum Reprod Update 2014;20:217-30.

10. Togashi K, Nishimura K, Kimura I, Tsuda Y, Yamashita K, Shibata T, et al. Endometrial cysts: diagnosis with MR imaging. Radiology 1991;180:73-8.

11. Herlidou-Même S, Constans JM, Carsin B, Olivie D, Eliat PA, Nadal-Desbarats L, et al. MRI texture analysis on texture test objects, normal brain and intracranial tumors. Magn Reson Im- 
aging 2003;21:989-93.

12. Bazot M, Bharwani N, Huchon C, Kinkel K, Cunha TM, Guerra A, et al. European society of urogenital radiology (ESUR) guidelines: MR imaging of pelvic endometriosis. Eur Radiol 2017;27:2765-75.

13. Forstner R, Meissnitzer MW, Schlattau A, Spencer JA. MRI in ovarian cancer. Imaging Med 2012;4:59-75.

14. Hornstein MD, Gleason RE, Orav J, Haas ST, Friedman AJ, Rein MS, et al. The reproducibility of the revised American Fertility Society classification of endometriosis. Fertil Steril 1993; 59:1015-21.

15. Schultes G. Klassifikation der Endometriose [Classification of endometriosis]. Wien Med Wochenschr 1999;149:361-5.

16. Lee HJ. Usefulness of subtraction pelvic magnetic resonance imaging for detection of ovarian endometriosis. Yeungnam Univ J Med 2020;37:90-7.

17. Grammatikakis I, Evangelinakis N, Salamalekis G, Tziortzioti V, Samaras C, Chrelias C, et al. Prevalence of severe pelvic inflammatory disease and endometriotic ovarian cysts: a 7-year retro- spective study. Clin Exp Obstet Gynecol 2009;36:235-6.

18. Mathias JM, Tofts PS, Losseff NA. Texture analysis of spinal cord pathology in multiple sclerosis. Magn Reson Med 1999; 42:929-35.

19. Castellano G, Bonilha L, Li LM, Cendes F. Texture analysis of medical images. Clin Radiol 2004;59:1061-9.

20. Lupean RA, Ștefan PA, Csutak C, Lebovici A, Măluțan AM, Buiga R, et al. Differentiation of endometriomas from ovarian hemorrhagic cysts at magnetic resonance: the role of texture analysis. Medicina (Kaunas) 2020;56:487.

21. Chan JH, Peh WC, Tsui EY, Wong KP, Yuen MK. Three-dimensional time-of-flight subtraction angiography of subacute cerebral hemorrhage. AJR Am J Roentgenol 2003;181:242-4.

22. Cheng B, Cai W, Sun C, Kang Y, Gong J. 3D bone subtraction $\mathrm{CT}$ angiography for the evaluation of intracranial aneurysms: a comparison study with $2 \mathrm{D}$ bone subtraction $\mathrm{CT}$ angiography and conventional non-subtracted CT angiography. Acta Radiol 2015;56:1127-34. 